\title{
Short-range magnetic correlations and spin dynamics in the paramagnetic regime of $(\mathrm{Mn}, \mathrm{Fe})_{2}(\mathrm{P}, \mathrm{Si})$
}

\author{
X. F. Miao,,${ }^{1, *}$ L. Caron,${ }^{1,2}$ J. Cedervall, ${ }^{3}$ P. C. M. Gubbens, ${ }^{1}$ P. Dalmas de Réotier, ${ }^{4,5}$ A. Yaouanc, ${ }^{4,5}$ \\ F. Qian, ${ }^{6}$ A. R. Wildes, ${ }^{7}$ H. Luetkens, ${ }^{8}$ A. Amato,${ }^{8}$ N. H. van Dijk, ${ }^{1}$ and E. Brück ${ }^{1}$ \\ ${ }^{1}$ Fundamental Aspects of Materials and Energy, Faculty of Applied Sciences, Delft University of Technology, \\ Mekelweg 15, $2629 \mathrm{JB}$ Delft, The Netherlands \\ ${ }^{2}$ Max Planck Institute for Chemical Physics of Solids, Nöthnitzer Straße 40, D-01187 Dresden, Germany \\ ${ }^{3}$ Department of Chemistry - Ångström Laboratory, Uppsala University, Box 538, 75121 Uppsala, Sweden \\ ${ }^{4}$ Université Grenoble Alpes, INAC-PHELIQS, F-38000 Grenoble, France \\ ${ }^{5}$ CEA, INAC-PHELIQS, F-38000 Grenoble, France \\ ${ }^{6}$ Neutron and Positron Methods in Materials, Faculty of Applied Sciences, Delft University of Technology, \\ Mekelweg 15, 2629 JB Delft, The Netherlands \\ ${ }^{7}$ Institut Laue-Langevin, 156X, 38042 Grenoble Cedex, France \\ ${ }^{8}$ Laboratory for Muon Spin Spectroscopy, Paul Scherrer Institute, 5232 Villigen-PSI, Switzerland
}

(Received 28 April 2016; revised manuscript received 9 June 2016; published 20 July 2016)

\begin{abstract}
The spatial and temporal correlations of magnetic moments in the paramagnetic regime of $(\mathrm{Mn}, \mathrm{Fe})_{2}(\mathrm{P}, \mathrm{Si})$ have been investigated by means of polarized neutron diffraction and muon-spin relaxation techniques. Shortrange magnetic correlations are present at temperatures far above the ferromagnetic transition temperature $\left(T_{C}\right)$. This leads to deviations of paramagnetic susceptibility from Curie-Weiss behavior. These short-range magnetic correlations extend in space, slow down with decreasing temperature, and finally develop into long-range magnetic order at $T_{C}$.
\end{abstract}

DOI: 10.1103/PhysRevB.94.014426

\section{INTRODUCTION}

The ferromagnetic transition in $(\mathrm{Mn}, \mathrm{Fe})_{2}(\mathrm{P}, \mathrm{Si})$ compounds is tied to a structure change due to a strong magnetoelastic coupling without altering the symmetry [1]. A giant magnetocaloric effect (GMCE) is accompanied with the magnetoelastic transition, which makes this material system promising for refrigeration and power conversion applications [2,3]. By balancing the Mn:Fe and P:Si ratios [2], the GMCE can be achieved in a wide composition range, to cover an extensive range of working temperatures required for real applications.

The $(\mathrm{Mn}, \mathrm{Fe})_{2}(\mathrm{P}, \mathrm{Si})$ compound crystallizes in a hexagonal $\mathrm{Fe}_{2} \mathrm{P}$-type structure [1] (space group $P \overline{6} 2 \mathrm{~m}$ ), as illustrated in Fig. 1. Fe atoms preferentially occupy the tetrahedral $3 f$ site, while Mn prefers the pyramidal $3 g$ site $[1,4]$. The nature of the ferromagnetic (FM) state, e.g., the magnitude and orientation of the magnetic moments, has been studied intensively using neutron diffraction techniques [1,5-7]. As shown in Fig. 1, both the Mn and Fe moments lie within the basal $a b$ plane in the FM phase.

A unique case of mixed magnetism has been revealed in the $(\mathrm{Mn}, \mathrm{Fe})_{2}(\mathrm{P}, \mathrm{Si})$ compound by density functional theory (DFT) calculations [2]. Above the ferromagnetic transition temperature $T_{C}$, the $\mathrm{Mn}$ atoms on the $3 g$ sites do not show a significant reduction in magnetic moment. In contrast, the Fe moment changes from a partially quenched value into a large value at the ferromagnetic transition. The DFT calculations [2] indicate that the $\mathrm{P}$ and $\mathrm{Si}$ atoms play a crucial role in the formation of a sizable magnetic moment for the $\mathrm{Fe}$ atoms. In the paramagnetic (PM) state, a covalent bond is formed

\footnotetext{
*x.f.miao@tudelft.nl
}

between the $\mathrm{Fe}$ and the neighboring $\mathrm{P}$ and $\mathrm{Si}$ atoms. As a result, the Fe moment is partially quenched. However, in the FM state, Fe carries a large magnetic moment instead of forming chemical bonds, due to the redistribution of electron density between the $\mathrm{Fe}$ and the $\mathrm{P}$ and $\mathrm{Si}$ atoms.

Magnetic measurements in the PM regime of (Mn,Fe) 2 (P, Si) compounds [7] show that the PM susceptibility strongly deviates from the Curie-Weiss law, which has also been observed in other $\mathrm{Fe}_{2} \mathrm{P}$-type compounds [8-10]. This suggests the presence of a sizable short-range magnetic order in the PM state of the $\mathrm{Fe}_{2} \mathrm{P}$-type compounds. Polarized-neutron diffraction studies on the $\mathrm{Fe}_{2} \mathrm{P}$ parent compound confirm the existence of short-range magnetic correlations above $T_{C}$ [11,12]. A magnetic correlation length of about $12.6 \AA$ was derived for $\mathrm{Fe}_{2} \mathrm{P}$ at $T=3.7 T_{C}$. Transverse-field muon-spin rotation and relaxation $(\mu \mathrm{SR})$ experiments on the $\mathrm{Fe}_{2} \mathrm{P}$ compound [13] reveal the absence of magnetic correlations with a lifetime longer than $10^{-10} \mathrm{~s}$ above a temperature of $1.05 T_{C}$. Compared to $\mathrm{Fe}_{2} \mathrm{P}$, stronger magnetic correlations are expected in the PM state of $(\mathrm{Mn}, \mathrm{Fe})_{2}(\mathrm{P}, \mathrm{Si})$ due to the larger magnetic moment of $\mathrm{Mn}$ on the $3 g$ site [2,14].

However, how the unusual transition in Fe moment develops from short-range magnetic correlations is still unknown and not accessible by DFT calculations. Also, the influence of short-range magnetic correlations on the nature of the transition is not well understood. In the present work, we aim to characterize the development of shortrange magnetic correlations on both length- and timescales in the $\mathrm{PM}$ regime of the $(\mathrm{Mn}, \mathrm{Fe})_{2}(\mathrm{P}, \mathrm{Si})$ compounds with both first and second order phase transitions, and further explore their roles in the magnetoelastic transition. Two compositions, which show first- and second-order phase transitions, respectively, were selected for the current 


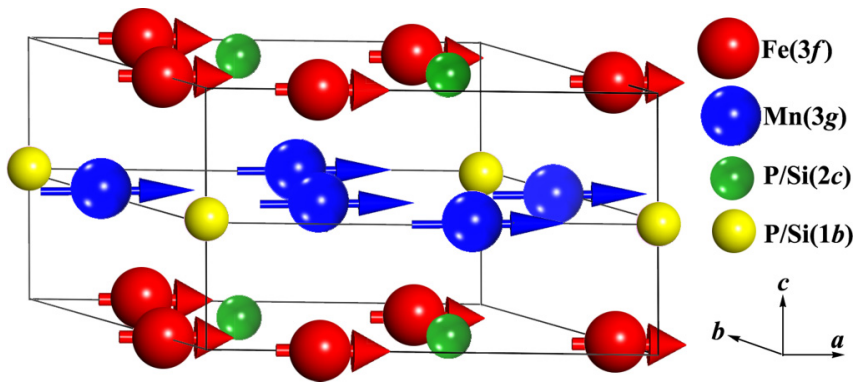

FIG. 1. Schematic representation of the crystal and magnetic structure for $(\mathrm{Mn}, \mathrm{Fe})_{2}(\mathrm{P}, \mathrm{Si})$.

study according to the previously derived compositional map [2].

We performed $x y z$ neutron polarization analysis [15-17] in the PM regime of the $(\mathrm{Mn}, \mathrm{Fe})_{2}(\mathrm{P}, \mathrm{Si})$ compounds. The unambiguous separation of the magnetic scattering cross section from the nuclear and spin-incoherent contributions allows us to characterize the spatial correlations of magnetic spins in the PM state. Zero-field muon-spin relaxation experiments were used to study the dynamics of the spin correlations in the PM regime. Consequently, this study contributes to a better understanding of the magnetoelastic phase-transition on both length- and timescales.

\section{EXPERIMENTAL}

Polycrystalline samples with nominal compositions of $\mathrm{Mn}_{1.00} \mathrm{Fe}_{0.95} \mathrm{P}_{0.67} \mathrm{Si}_{0.33}$ and $\mathrm{Mn}_{1.70} \mathrm{Fe}_{0.25} \mathrm{P}_{0.50} \mathrm{Si}_{0.50}$ were prepared as described previously [2]. AC susceptibility measurements were performed on a superconducting quantum interference device (SQUID) magnetometer (Quantum Design MPMS $5 \mathrm{XL}$ ). The amplitude and frequency of the AC magnetic field were $0.4 \mathrm{mT}$ and $111 \mathrm{~Hz}$, respectively. No DC field was applied during the AC susceptibility measurements. X-ray diffraction patterns were collected on a Bruker D8 diffractometer using $\mathrm{Cu} K_{\alpha 1}$ radiation. Structure refinement of the X-ray diffraction patterns was performed using Fullprof's [18] implementation of the Rietveld refinement method.

$x y z$ neutron polarization experiments were performed on the D7 diffuse scattering diffractometer [16] at the Institut Laue-Langevin (ILL), with an incident neutron wavelength of $3.12 \AA$. The powder samples $(\approx 10$ grams) were put into an aluminum hollow cylinder. Six spin-dependent scattering cross sections were measured for $x y z$ polarization analysis between $150 \mathrm{~K} \leq T \leq 500 \mathrm{~K}$. The scattering cross sections were integrated for energy transfers ranging from about -10 to $8.5 \mathrm{meV}$. The instrument-dependent background was estimated from measurements of an empty sample can and a cadmium sample. Amorphous quartz was measured to establish the polarization efficiency for D7. Vanadium was measured to calibrate the detector efficiencies and to allow the scattering cross sections from the samples to be expressed in absolute units.

$\mu$ SR experiments were carried out on the general purpose surface-muon instrument (GPS) at the Swiss muon source $(\mathrm{S} \mu \mathrm{S})$ of the Paul Scherrer Institute (PSI), Switzerland. Zerofield muon-spin relaxation signals were collected for powder samples $(\approx 1$ gram $)$ in the temperature range between 50 and $450 \mathrm{~K}$ using a closed cycle refrigerator.

\section{RESULTS}

\section{A. Magnetic susceptibility and x-ray diffraction}

As shown in Fig. 2(a), the $\mathrm{Mn}_{1.00} \mathrm{Fe}_{0.95} \mathrm{P}_{0.67} \mathrm{Si}_{0.33}$ compound shows a large thermal hysteresis in the bulk susceptibility measurements, which is characteristic for a strong first-order phase transition. In contrast, the reversible PM-FM transition in Fig. 2(b) for the $\mathrm{Mn}_{1.70} \mathrm{Fe}_{0.25} \mathrm{P}_{0.50} \mathrm{Si}_{0.50}$ compound suggests a second-order nature of the phase transition, in agreement with our previous work [19]. The $T_{C}$ values [20] on cooling for the $\mathrm{Mn}_{1.00} \mathrm{Fe}_{0.95} \mathrm{P}_{0.67} \mathrm{Si}_{0.33}$ and $\mathrm{Mn}_{1.70} \mathrm{Fe}_{0.25} \mathrm{P}_{0.50} \mathrm{Si}_{0.50}$ compounds are 130 and $175 \mathrm{~K}$, respectively. Additionally, the $\mathrm{Mn}_{1.00} \mathrm{Fe}_{0.95} \mathrm{P}_{0.67} \mathrm{Si}_{0.33}$ compound shows an antiferromagnetic (AFM) transition at a Néel temperature of $T_{N} \approx 275 \mathrm{~K}$ [see the inset of Fig. 2(a)]. The absence of thermal hysteresis implies that this PM-AFM transition is second order. It should be noted that the AFM intermediate phase has only been found in certain $(\mathrm{Mn}, \mathrm{Fe})_{2}(\mathrm{P}, \mathrm{Si})$ compositions [21]. The formation of the AFM phase is due to the competing magnetic configurations and strong magnetoelastic coupling in $(\mathrm{Mn}, \mathrm{Fe})_{2}(\mathrm{P}, \mathrm{Si})$ compounds, as revealed by recent theoretical [22] and experimental [21] studies.

The inverse susceptibility in Figs. 2(c) and 2(d) measured above $T_{C}$ for the $\mathrm{Mn}_{1.00} \mathrm{Fe}_{0.95} \mathrm{P}_{0.67} \mathrm{Si}_{0.33}$ and $\mathrm{Mn}_{1.70} \mathrm{Fe}_{0.25} \mathrm{P}_{0.50} \mathrm{Si}_{0.50}$ compounds, respectively, deviates from the Curie-Weiss law. This reflects the presence and development of short-range magnetic correlations in the PM state [7-10], which was confirmed by our neutron (Sec. III B) and muon experiments (Sec. III C).

Figure 3 shows the contour plots of the x-ray diffraction patterns measured on cooling for the two compounds. The discontinuous and continuous shift in the peak position manifests the first- and second-order phase transition in the $\mathrm{Mn}_{1.00} \mathrm{Fe}_{0.95} \mathrm{P}_{0.67} \mathrm{Si}_{0.33}$ and $\mathrm{Mn}_{1.70} \mathrm{Fe}_{0.25} \mathrm{P}_{0.50} \mathrm{Si}_{0.50}$ compounds, respectively. The coincidence of structural (see Fig. 3) and magnetic (see Fig. 2) transitions characterizes the strong
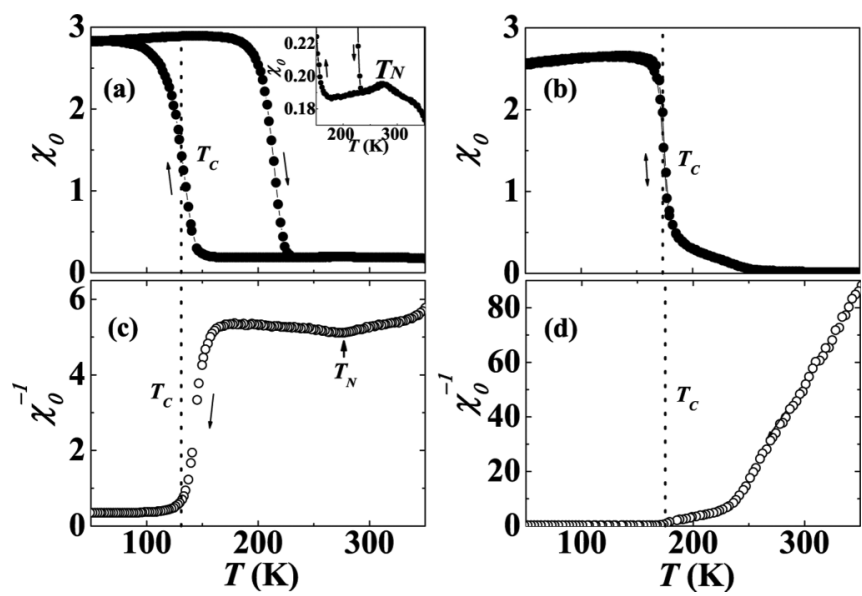

FIG. 2. Bulk magnetic susceptibility (unitless) for $\mathrm{Mn}_{1.00} \mathrm{Fe}_{0.95} \mathrm{P}_{0.67} \mathrm{Si}_{0.33}$ (a) and $\mathrm{Mn}_{1.70} \mathrm{Fe}_{0.25} \mathrm{P}_{0.50} \mathrm{Si}_{0.50}$ (b). The corresponding inverse susceptibility is shown in (c) and (d), respectively. 

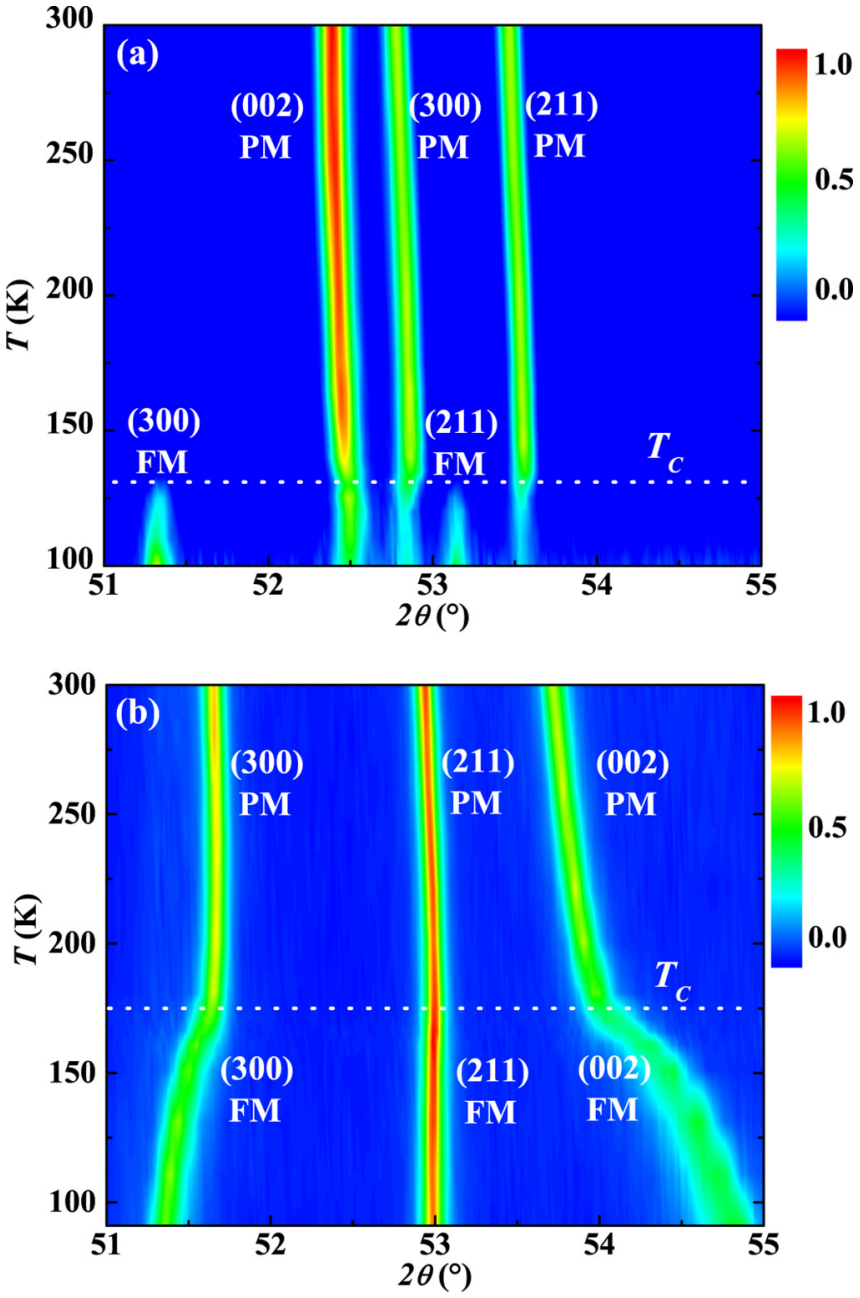

FIG. 3. Contour plots of the $\mathrm{x}$-ray diffraction patterns measured on cooling for $\mathrm{Mn}_{1.00} \mathrm{Fe}_{0.95} \mathrm{P}_{0.67} \mathrm{Si}_{0.33}$ (a) and $\mathrm{Mn}_{1.70} \mathrm{Fe}_{0.25} \mathrm{P}_{0.50} \mathrm{Si}_{0.50}$ (b). The color bar on the right represents the normalized intensity scale.

magnetoelastic coupling in $(\mathrm{Mn}, \mathrm{Fe})_{2}(\mathrm{P}, \mathrm{Si})$ compounds. The lattice parameters derived from Rietveld refinement are presented in Fig. 4. The anomalous thermal evolution of the lattice parameters close to $T_{C}$ in the PM regime, which is a common feature in $(\mathrm{Mn}, \mathrm{Fe})_{2}(\mathrm{P}, \mathrm{Si})$ compounds [7,23], reflects the development of magnetic correlations.

\section{B. Neutron polarization analysis}

Polarized neutron scattering experiments were performed in the PM regime of the $\mathrm{Mn}_{1.00} \mathrm{Fe}_{0.95} \mathrm{P}_{0.67} \mathrm{Si}_{0.33}$ and $\mathrm{Mn}_{1.70} \mathrm{Fe}_{0.25} \mathrm{P}_{0.50} \mathrm{Si}_{0.50}$ compounds. Figure 5(a) shows the separate magnetic and nuclear scattering cross section at $500 \mathrm{~K}$ for the $\mathrm{Mn}_{1.00} \mathrm{Fe}_{0.95} \mathrm{P}_{0.67} \mathrm{Si}_{0.33}$ compound. The clear forward (low- $Q$ ) magnetic scattering indicates the presence of weak short-range FM correlations at $500 \mathrm{~K}$ (about $3.8 T_{C}$ ) [12,24]. The magnetic scattering cross section measured at $175 \mathrm{~K}$ [see Fig. 5(b)] shows a weak peak at $Q \approx 0.4 \AA^{-1}$. A similar neutron-diffraction peak has been observed in certain $(\mathrm{Mn}, \mathrm{Fe})_{2}(\mathrm{P}, \mathrm{Si})$ compositions, originating from weak incommensurate AFM order $[21,24]$. The nuclear scattering cross
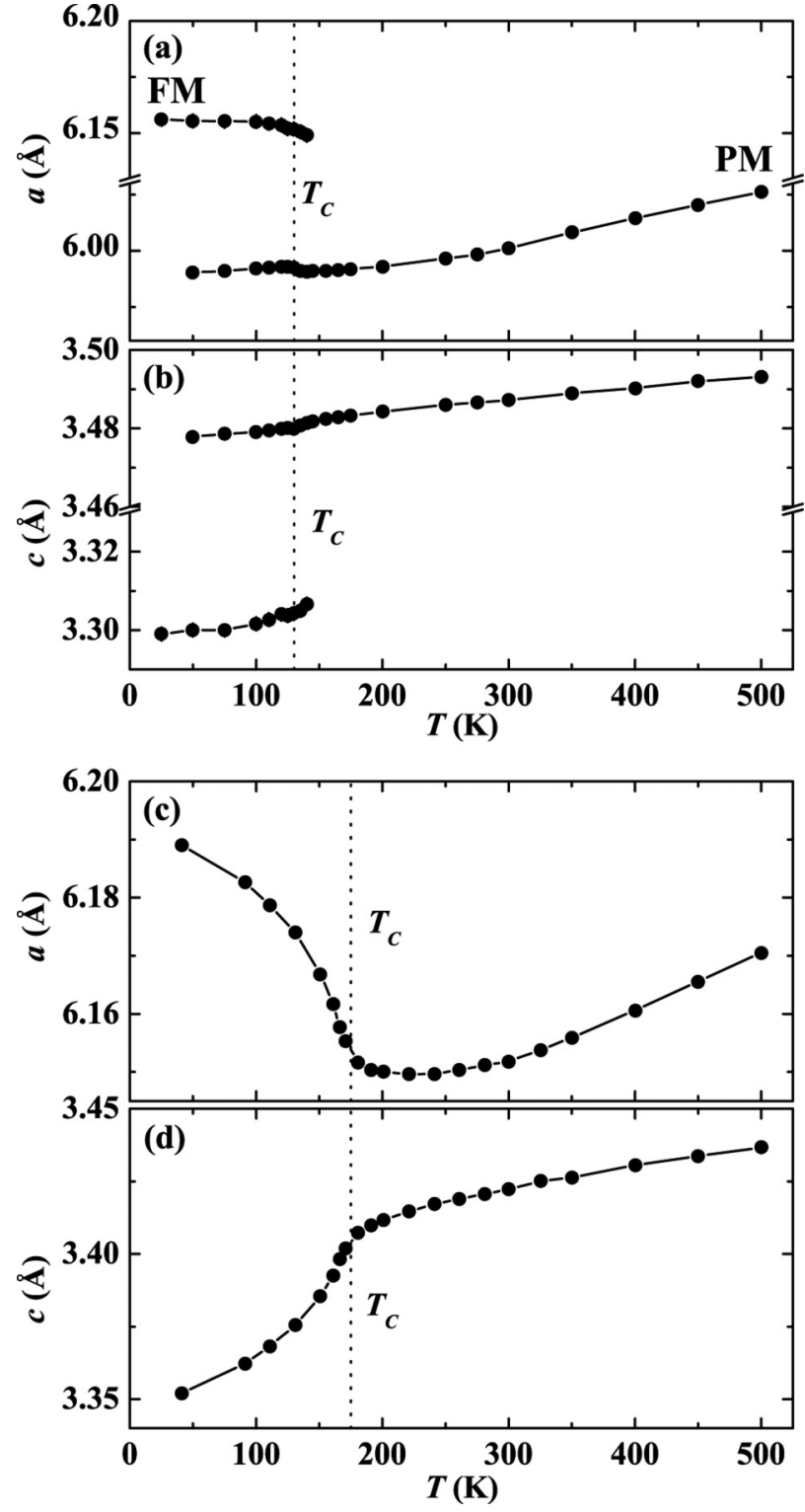

FIG. 4. Lattice parameters derived from $\mathrm{x}$-ray diffraction for $\mathrm{Mn}_{1.00} \mathrm{Fe}_{0.95} \mathrm{P}_{0.67} \mathrm{Si}_{0.33}$ (a),(b) and $\mathrm{Mn}_{1.70} \mathrm{Fe}_{0.25} \mathrm{P}_{0.50} \mathrm{Si}_{0.50}$ (c),(d).

section shows slight changes in the peak positions with decreasing temperature due to thermal expansion.

In order to explore the evolution of magnetic correlations in detail for the $\mathrm{Mn}_{1.00} \mathrm{Fe}_{0.95} \mathrm{P}_{0.67} \mathrm{Si}_{0.33}$ compound, the magnetic scattering cross section is plotted at different temperatures upon cooling in Fig. 6. The forward scattering becomes more pronounced when the sample is cooled from 500 to $350 \mathrm{~K}$, which is due to the development of magnetic correlations. However, AFM order $[21,24]$ appears at $275 \mathrm{~K}$, as suggested by the weak peak at $Q \approx 0.36 \AA^{-1}$. With a further decrease in temperature, the peak intensity significantly increases and the peak position shifts to $\operatorname{larger} Q$ values. This reveals the enhancement of AFM order and variations in the propagation vector of the incommensurate AFM structure [21]. The detected AFM order is consistent with the susceptibility measurements [see the inset of Fig. 2 (a)] and previous neutron diffraction experiments [21]. Intensive temperature-dependent 


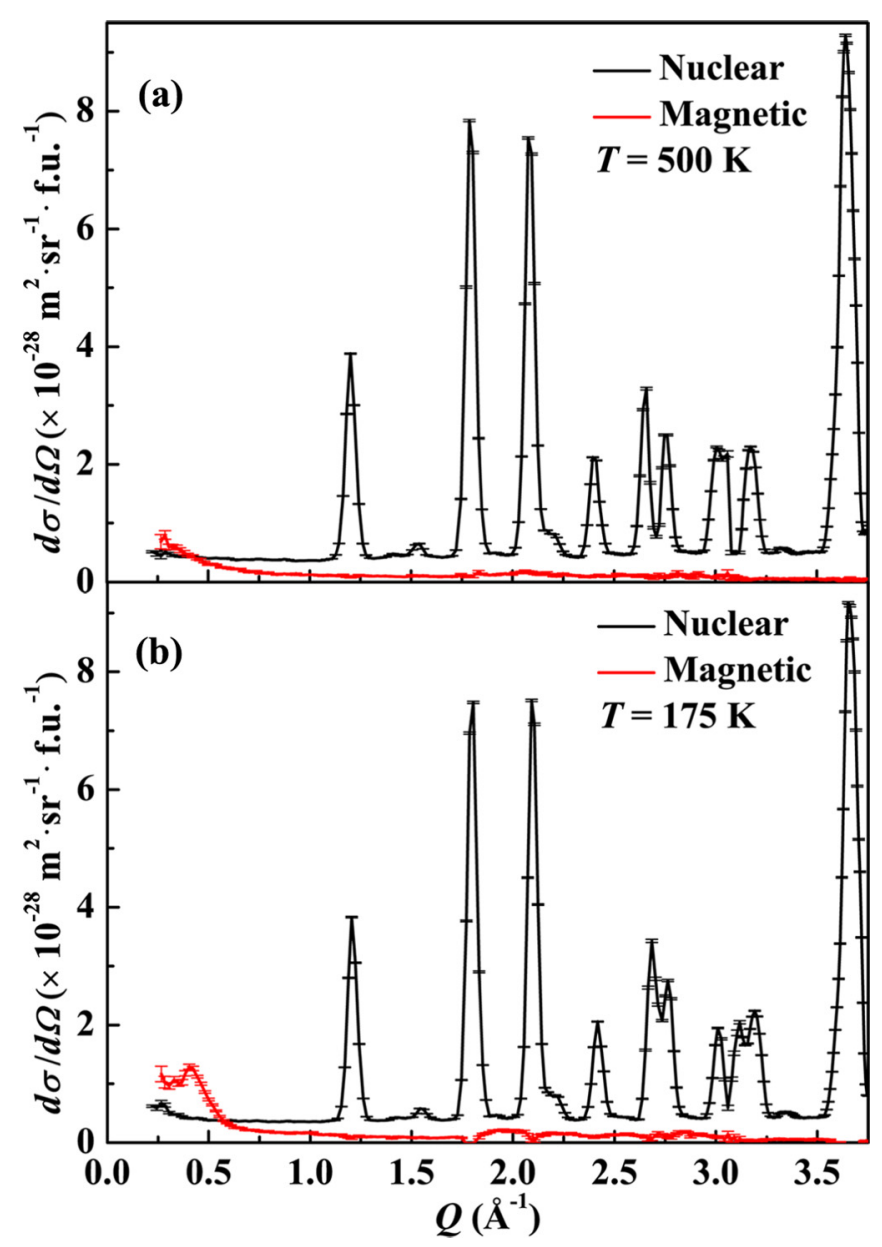

FIG. 5. Magnetic and nuclear scattering cross section for $\mathrm{Mn}_{1.00} \mathrm{Fe}_{0.95} \mathrm{P}_{0.67} \mathrm{Si}_{0.33}$ measured at $500 \mathrm{~K}$ (a) and $175 \mathrm{~K}$ (b).

neutron diffraction measurements [21] clearly reveal that some $(\mathrm{Mn}, \mathrm{Fe})_{2}(\mathrm{P}, \mathrm{Si})$ compounds undergo a PM to AFM phase transition before further transforming into a FM phase upon cooling.

In contrast to the $\mathrm{Mn}_{1.00} \mathrm{Fe}_{0.95} \mathrm{P}_{0.67} \mathrm{Si}_{0.33}$ compound, only FM correlations are detected in the $\mathrm{Mn}_{1.70} \mathrm{Fe}_{0.25} \mathrm{P}_{0.50} \mathrm{Si}_{0.50}$

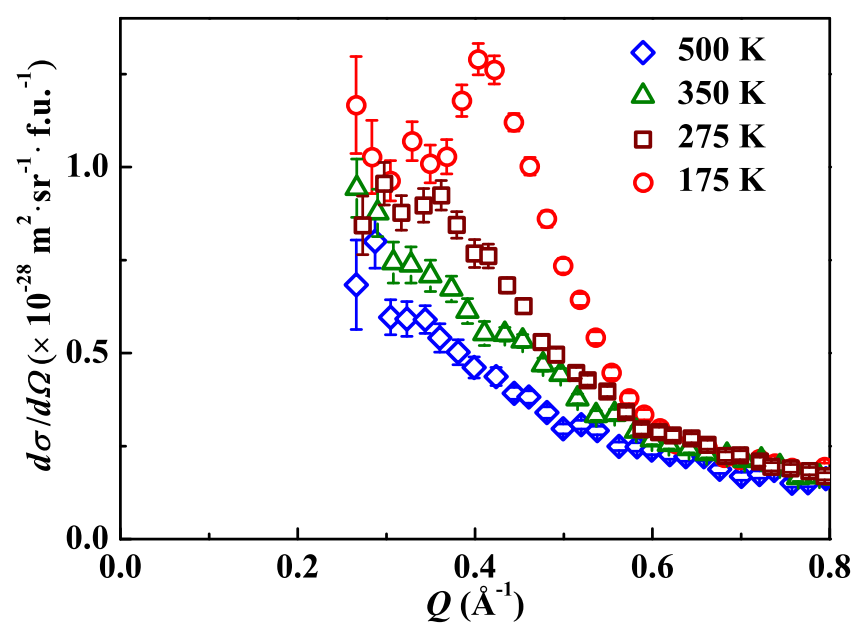

FIG. 6. Magnetic scattering cross section as a function of temperature for $\mathrm{Mn}_{1.00} \mathrm{Fe}_{0.95} \mathrm{P}_{0.67} \mathrm{Si}_{0.33}$.

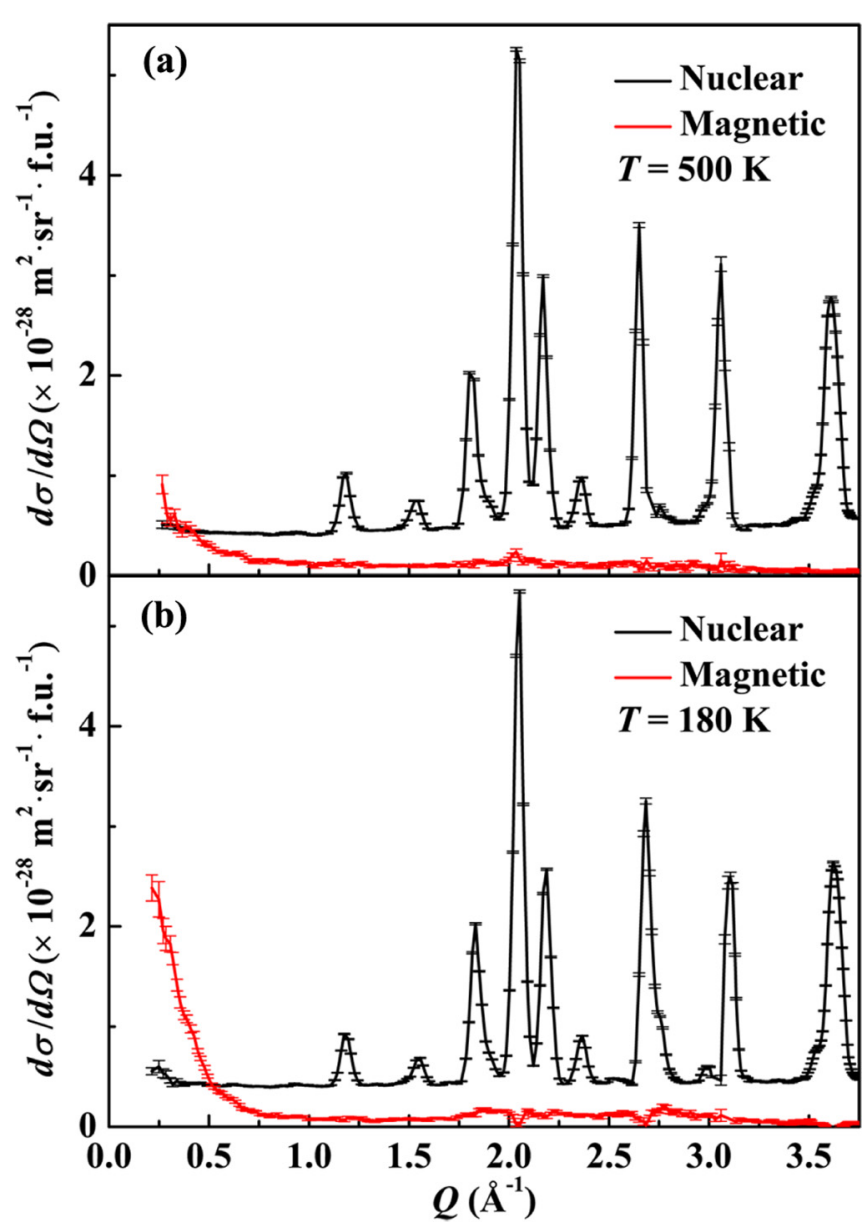

FIG. 7. Magnetic and nuclear scattering cross section for $\mathrm{Mn}_{1.70} \mathrm{Fe}_{0.25} \mathrm{P}_{0.50} \mathrm{Si}_{0.50}$ measured at $500 \mathrm{~K}$ (a) and $180 \mathrm{~K}$ (b).

compound at 500 and $180 \mathrm{~K}$, as indicated by the forward neutron diffuse scattering [24] in Figs. 7(a) and 7(b). The FM correlations are enhanced with decreasing temperature, manifested by the increasing intensity of the forward scattering in Fig. 8(a). We assume that scattering cross sections outside the detection energy window of the D7 diffractometer are negligible. The magnetic cross section in the vicinity of $Q=0$ for a paramagnetic phase is approximately given by [25]

$$
\frac{d \sigma}{d \Omega}(Q)=\frac{2}{3} N\left(\frac{g_{n} r_{0}}{4 \mu_{B}}\right)^{2} \frac{k_{B} T M}{\rho N_{A} \mu_{0}} \chi(Q)
$$

where $N$ is the number of magnetic atoms per formula unit, $g_{n}$ is the neutron $g$ factor, $r_{0}$ is the classical electron radius, $\mu_{B}$ is the Bohr magneton, $k_{B}$ is the Boltzmann constant, $M$ is the molar mass, $\rho$ is the volumetric-mass density, $N_{A}$ is the Avogadro constant, $\mu_{0}$ is the permeability of vacuum, and $\chi(Q)$ is the magnetic susceptibility. We will thereafter assume that the $Q$ dependence of $\chi(Q)$ is described by a Lorentzian function. The $\chi(Q \rightarrow 0)$ equals the bulk magnetic susceptibility, i.e., the $\chi_{0}$ shown in Fig. 2. As a result, the $\frac{d \sigma}{d \Omega}(0)$ at different temperatures can be calculated from $\chi_{0}$ using Eq. (1).

The forward scattering cross section in Fig. 8(a) can be fitted well with the convolution of a Gaussian (instrument resolution [26,27] with a full width at half maximum of about $0.06 \AA^{-1}$ in the vicinity of $Q=0$ ) and the Lorentzian (sample 

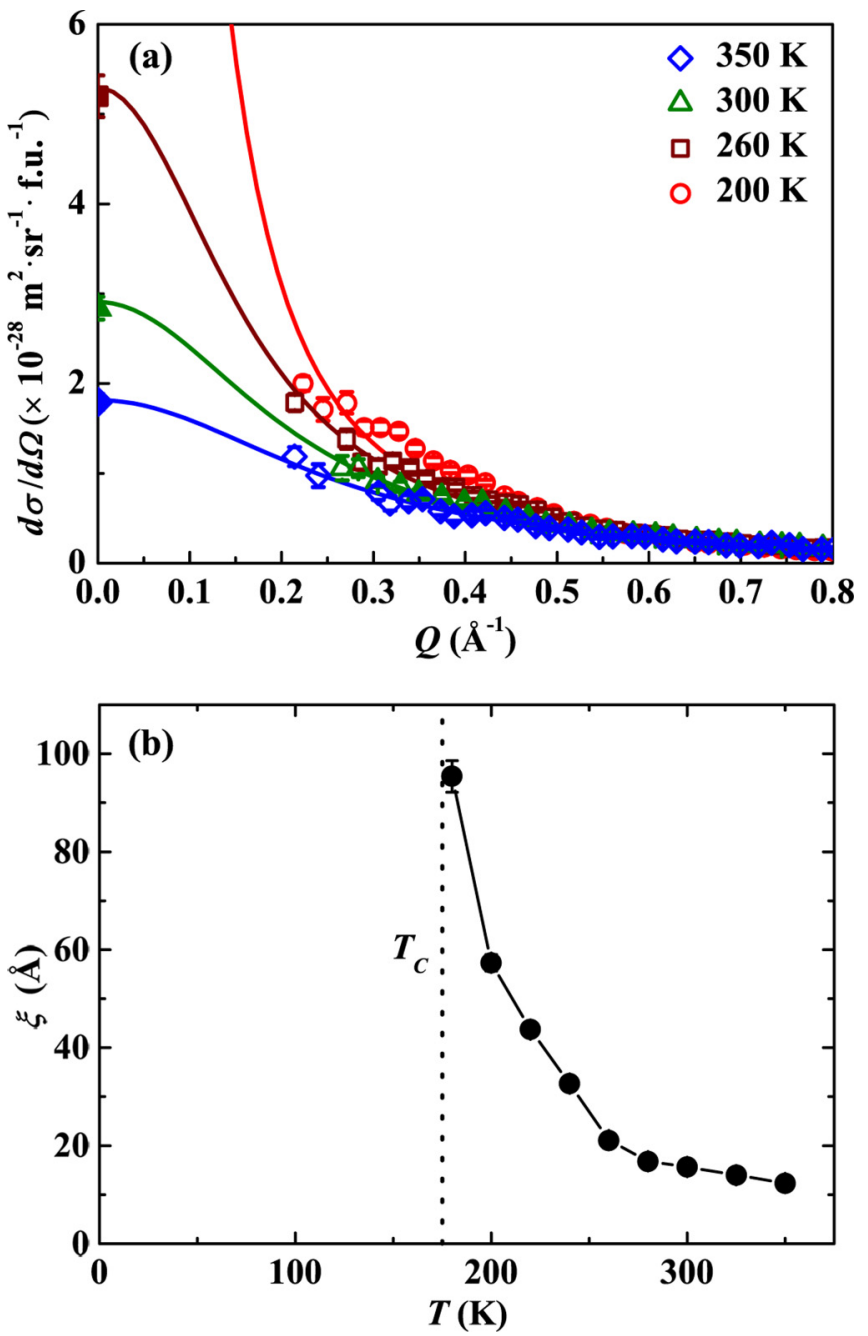

FIG. 8. Magnetic scattering cross section (a) and the derived magnetic correlation length (b) as a function of temperature for $\mathrm{Mn}_{1.70} \mathrm{Fe}_{0.25} \mathrm{P}_{0.50} \mathrm{Si}_{0.50}$. The solid lines in (a) represent fits to the data.

contribution) function. From the full width at half maximum $\Delta Q$ of the Lorentzian function, the magnetic correlation length $\xi=2 \pi / \Delta Q$ can be derived at different temperatures. As shown in Fig. 8(b), at $350 \mathrm{~K}\left(=2.0 T_{C}\right)$, the FM correlation length in the $\mathrm{Mn}_{1.70} \mathrm{Fe}_{0.25} \mathrm{P}_{0.50} \mathrm{Si}_{0.50}$ compound is $12.4 \AA$, which is comparable with $\xi=13.0 \AA$ observed at $T=2.1 T_{C}$ for the $\mathrm{Fe}_{2} \mathrm{P}$ compound [12]. The value of $\xi$ rises with the decrease in temperature and reaches $31.6 \AA$ at $240 \mathrm{~K}$ (= $1.4 T_{C}$ ) for the $\mathrm{Mn}_{1.70} \mathrm{Fe}_{0.25} \mathrm{P}_{0.50} \mathrm{Si}_{0.50}$ compound, which is much larger than the $\xi=16.6 \AA$ observed at $T=1.4 T_{C}$ for the $\mathrm{Fe}_{2} \mathrm{P}$ compound [12]. The correlation length further increases to $95.8 \AA$ at $180 \mathrm{~K}$ in the $\mathrm{Mn}_{1.70} \mathrm{Fe}_{0.25} \mathrm{P}_{0.50} \mathrm{Si}_{0.50}$ compound. The thermal evolution of the correlation length provides clear evidence for the spatial development of magnetic correlations in the $\mathrm{PM}$ regime for $(\mathrm{Mn}, \mathrm{Fe})_{2}(\mathrm{P}, \mathrm{Si})$ compounds.

\section{Muon-spin relaxation analysis}

The dynamics of the magnetic correlations above $T_{C}$ was studied by means of muon-spin relaxation experiments for the two compounds. In the zero-field $\mu \mathrm{SR}$ geometry, when the strength of the static local magnetic field is assumed to show a Gaussian distribution, the muon-spin relaxation function is described by the so-called Kubo-Toyabe function [28,29]

$$
A^{\text {stat }}(t)=A_{0}\left[\frac{1}{3}+\frac{2}{3}\left(1-\gamma_{\mu}^{2} \Delta^{2} t^{2}\right) \exp \left(\frac{-\gamma_{\mu}^{2} \Delta^{2} t^{2}}{2}\right)\right],
$$

where $A_{0}$ is the initial asymmetry, $\gamma_{\mu}$ is the gyromagnetic ratio of the muon $\left(8.51616 \times 10^{8} \mathrm{rad} \mathrm{s}^{-1} \mathrm{~T}^{-1}\right)$, and $\Delta$ is the standard deviation of the Gaussian field distribution.

Muon diffusion and the fluctuations of the local field will cause dynamics of the muon-spin relaxation. This can be evaluated within the framework of the strong collision model [29-32]. In the slow dynamic limit, the muon-spin relaxation is derived as [29-32]

$$
\begin{aligned}
A^{\text {dyn }}(t)= & A_{0}\left[\frac{1}{3} \exp \left(-\frac{2}{3} v t\right)\right. \\
& \left.+\frac{2}{3}\left(1-\gamma_{\mu}^{2} \Delta^{2} t^{2}\right) \exp \left(\frac{-\gamma_{\mu}^{2} \Delta^{2} t^{2}}{2}\right)\right],
\end{aligned}
$$

where $v$ is the local-field correlation frequency due to the muon diffusion and the local-field fluctuations. In the fast dynamic limit, the muon-spin relaxation function is written as [29-32]

$$
A^{\mathrm{dyn}}(t)=A_{0} \exp (-\lambda t),
$$

where $\lambda$ is the muon-spin relaxation rate.

Considering the large nuclear magnetic moment of $\mathrm{Mn}$ and $\mathrm{P}$, nuclear magnetic fields as well as the magnetic fields due to the unpaired electrons of $\mathrm{Mn}$ and $\mathrm{Fe}$ are probed by the implanted muons. Muon diffusion will cause dynamics for the nuclear contribution. As a first approximation, we assume a slow-dynamics behavior for the nuclear contribution, which is described by Eq. (3). As for the muon-spin relaxation of electronic origin, its dynamics can be caused by the fluctuations of the magnetic correlations. The electronic contribution above $T_{C}$ is expected to be dominated by fast dynamics, which is described by Eq. (4). The nuclear and electronic contributions are independent, and hence the zero-field $\mu \mathrm{SR}$ spectra measured above $T_{C}$ are fitted by a product of Eqs. (3) and (4). Fits for both compounds show that muon diffusion is present above $325 \mathrm{~K}$, while it becomes negligible below $325 \mathrm{~K}$. Muon diffusion is a thermally-activated process. Its contribution therefore follows an Arrhenius law. As a result, this contribution decreases rapidly with a decrease in temperature and was found to be negligible below $325 \mathrm{~K}$. This indicates a static nuclear contribution below $325 \mathrm{~K}$, where the $\mu$ SR spectra can be fitted by a product of Eqs. (2) and (4). As a consequence, the fluctuations of the magnetic correlations can be unambiguously extracted from the zero-field $\mu$ SR spectra below $325 \mathrm{~K}$.

Figure 9 shows the zero-field $\mu \mathrm{SR}$ spectra measured below $325 \mathrm{~K}$ for $\mathrm{Mn}_{1.00} \mathrm{Fe}_{0.95} \mathrm{P}_{0.67} \mathrm{Si}_{0.33}$. Above $T_{C}(\approx 130 \mathrm{~K})$, the spectra show a fast damping behavior, suggesting dynamic magnetic fields experienced by the muons. Below $T_{C}$, the initial asymmetry in the spectrum drops to $1 / 3$ of that in the PM state without showing any oscillation signals. This reflects the broad distribution of local magnetic fields at different muon sites in the magnetically-ordered polycrystalline sample. The standard deviation of the nuclear field distribution $\Delta_{n}$ in Eq. (2) 


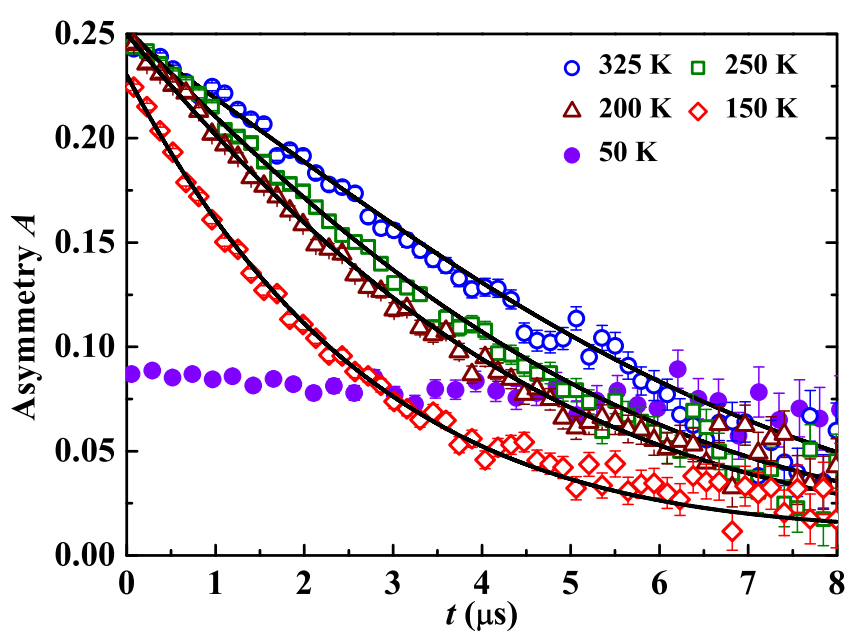

FIG. 9. Zero-field $\mu$ SR spectra for $\mathrm{Mn}_{1.00} \mathrm{Fe}_{0.95} \mathrm{P}_{0.67} \mathrm{Si}_{0.33}$. The solid lines represent fits to the data.

derived from the fits is about $0.13 \mathrm{mT}$. The initial asymmetry in Fig. 10(a) shows a decrease for temperatures close to $T_{C}$, which suggests that the sample is not magnetically homogeneous due to compositional inhomogeneity. There is also the possibility that the slowing down of the magnetic fluctuations is so strong that the motional narrowing limit assumed by Eq. (4) is no longer valid. The muon-spin relaxation rate, presented in Fig. 10(b), gradually increases as $T_{C}$ is approached from

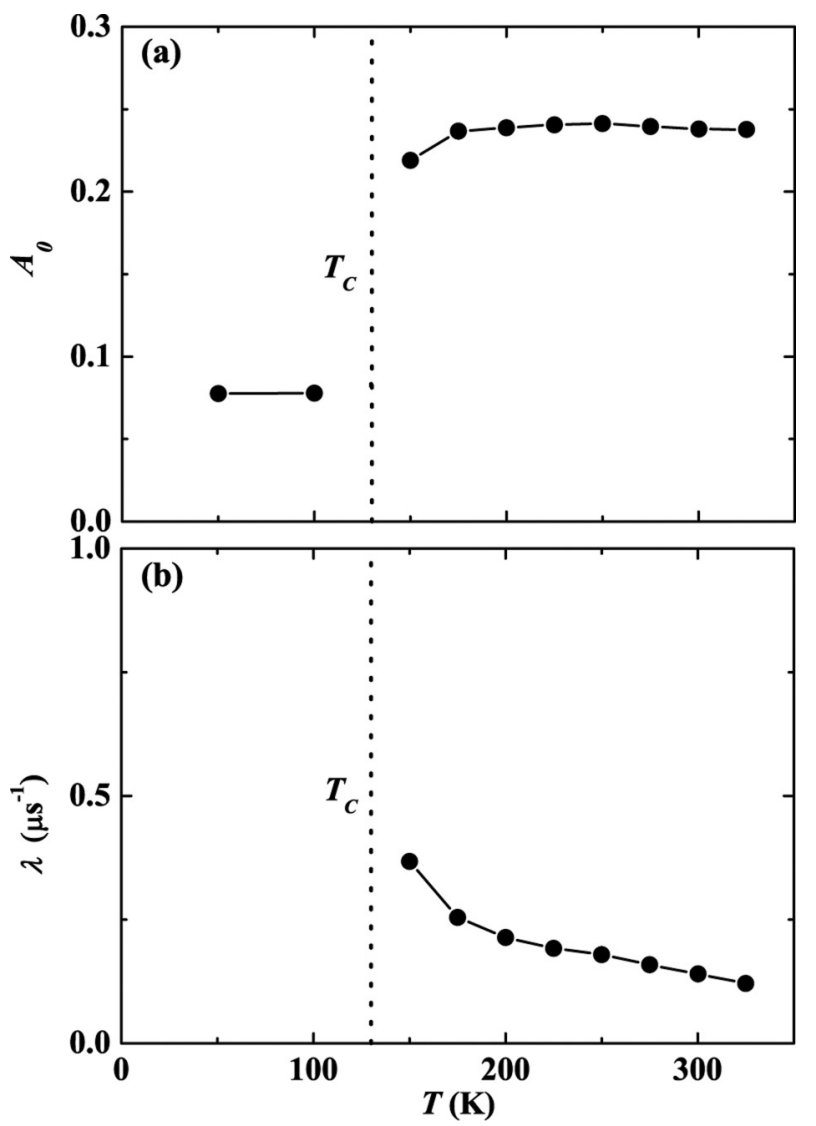

FIG. 10. Temperature dependence of initial asymmetry $A_{0}$ (a) and muon-spin relaxation rate $\lambda$ (b) for $\mathrm{Mn}_{1.00} \mathrm{Fe}_{0.95} \mathrm{P}_{0.67} \mathrm{Si}_{0.33}$.

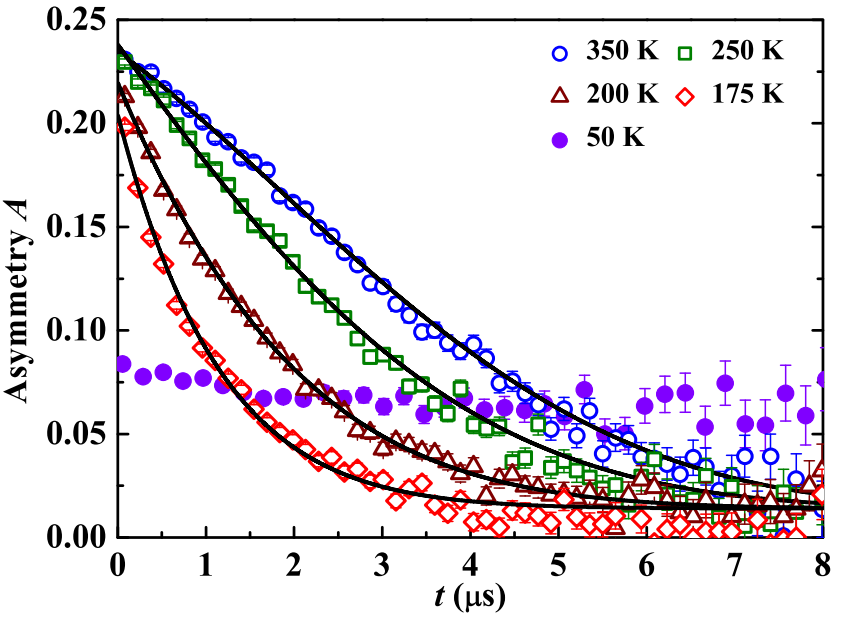

FIG. 11. Zero-field $\mu$ SR spectra for $\mathrm{Mn}_{1.70} \mathrm{Fe}_{0.25} \mathrm{P}_{0.50} \mathrm{Si}_{0.50}$. The solid lines represent fits to the data.

the high-temperature side. It should be noted that we did not observe a significant change in the muon results around the $T_{N} \approx 275 \mathrm{~K}$ for the $\mathrm{Mn}_{1.00} \mathrm{Fe}_{0.95} \mathrm{P}_{0.67} \mathrm{Si}_{0.33}$ sample. This is probably due to the fact that in the vicinity of $T_{N}$ the sample is still dominated by ferromagnetic short-range correlations with a small amount of AFM order. As a result, the muon spectra are mainly characterized by the fluctuations of the ferromagnetic correlations with a small contribution from the AFM phase.

The $\mathrm{Mn}_{1.70} \mathrm{Fe}_{0.25} \mathrm{P}_{0.50} \mathrm{Si}_{0.50}$ compound shows similar $\mu \mathrm{SR}$ signals (see Fig. 11) as $\mathrm{Mn}_{1.00} \mathrm{Fe}_{0.95} \mathrm{P}_{0.67} \mathrm{Si}_{0.33}$. The same fitting model is employed, and the derived parameters are presented in Fig. 12. The standard deviation of the nuclear field distribution $\Delta_{n}$ is about $0.21 \mathrm{mT}$ for the $\mathrm{Mn}_{1.70} \mathrm{Fe}_{0.25} \mathrm{P}_{0.50} \mathrm{Si}_{0.50}$ compound. As shown in Fig. 12(a), the initial asymmetry starts to decrease above $T_{C}$, which is similar to that for the $\mathrm{Mn}_{1.00} \mathrm{Fe}_{0.95} \mathrm{P}_{0.67} \mathrm{Si}_{0.33}$ compound. This may be caused by compositional inhomogeneity or indicating that our assumption of motional narrowing limit is no longer applicable. The muon-spin relaxation rate increases with decreasing temperature and tends to diverge close to $T_{C}$ [see Fig. 12(b)].

For isotropic ferromagnetic dynamical correlations with a well-developed maximum of $\chi(Q)$ at $Q=0$ and assuming a Lorentzian spectral-weight function, the muon-spin relaxation rate measured in zero magnetic field can be written as [32]

$$
\lambda=\frac{\mu_{0} \gamma_{\mu}^{2}}{\pi^{2}}\left[\left(p-\frac{1}{3}\right)^{2}+\frac{2}{9}\right] k_{B} T \int \chi(Q) \frac{Q^{2}}{\Gamma_{0}(Q)} d Q,
$$

where the $p$ is a constant that depends on the hyperfine constant and the number of neighboring ions coupled to the muon spin through the hyperfine field. As a first approximation, the value of $\left(p-\frac{1}{3}\right)^{2}+\frac{2}{9}$ is assumed to be 1 , taken as a realistic order of magnitude [32] for the materials studied here. $\Gamma_{0}(Q)$ is the magnetic fluctuation rate at wave vector $Q$.

Since short-range magnetic correlations are clearly observed from neutron diffraction experiments (see Fig. 8), $\Gamma_{0}(Q)$ should be dominated by spin-conserved dynamics $[32,33]$. We assume that $\hbar \Gamma_{0}(Q)=D_{s d} Q^{2}$, i.e., neglecting the high-order terms in $Q[32,33]$, where $D_{s d}$ is a proportionality factor. From Eqs. (1) and (5), the value of $\Gamma_{0}$ for the magnetic correlation with a correlation length of $\xi$ is derived 

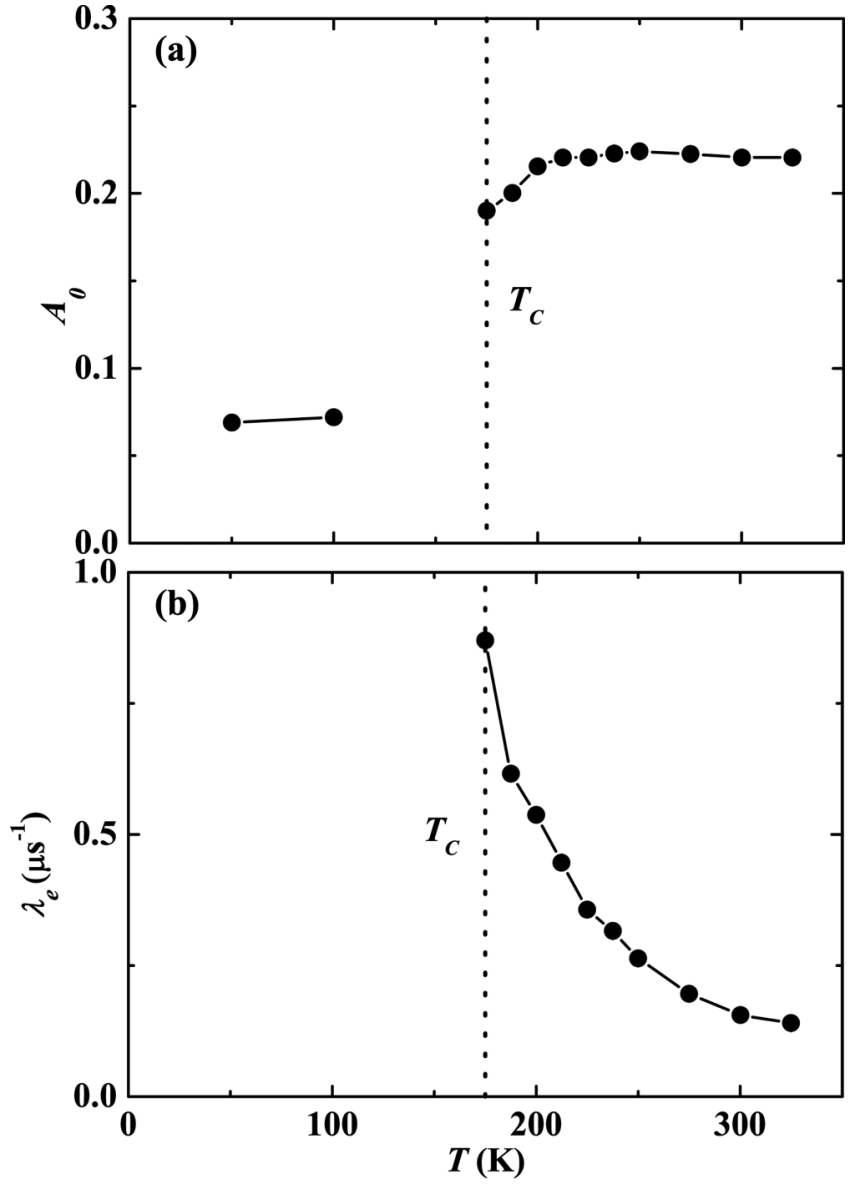

FIG. 12. Temperature dependence of initial asymmetry $A_{0}$ (a) and muon-spin relaxation rate $\lambda$ (b) for $\mathrm{Mn}_{1.70} \mathrm{Fe}_{0.25} \mathrm{P}_{0.50} \mathrm{Si}_{0.50}$.

as:

$$
\Gamma_{0}\left(\frac{2 \pi}{\xi}\right)=\frac{C}{\hbar} \frac{\int \frac{d \sigma}{d \Omega}(Q) d Q}{\lambda}\left(\frac{2 \pi}{\xi}\right)^{2},
$$

where $C=6.04 \times 10^{-24} \mathrm{~J} \mathrm{~m} \mathrm{~s}^{-1}$. The $\frac{d \sigma}{d \Omega}(Q), \quad \xi$ and $\lambda$ for the $\mathrm{Mn}_{1.70} \mathrm{Fe}_{0.25} \mathrm{P}_{0.50} \mathrm{Si}_{0.50}$ compound are presented in Figs. 8(a), 8(b) and 12(b), respectively. The magnetic relaxation rate $\Gamma_{0}$ is calculated at different temperatures and presented in Fig. 13. The magnetic relaxation rate decreases with decreasing temperature, which characterizes the slowing down of magnetic fluctuations in the $\mathrm{PM}$ regime of the $\mathrm{Mn}_{1.70} \mathrm{Fe}_{0.25} \mathrm{P}_{0.50} \mathrm{Si}_{0.50}$ compound when $T_{C}$ is approached. The magnetic correlation time $\tau=1 / \Gamma_{0}(2 \pi / \xi)$ is in the microsecond time range at temperatures $1.03 T_{C} \leq T \leq 1.86 T_{C}$ for the $\mathrm{Mn}_{1.70} \mathrm{Fe}_{0.25} \mathrm{P}_{0.50} \mathrm{Si}_{0.50}$ compound, while no correlations with $\tau$ longer than $10^{-10} \mathrm{~s}$ were detected at $T \geq 1.05 T_{C}$ for the $\mathrm{Fe}_{2} \mathrm{P}$ compound. This confirms stronger magnetic correlations in the $(\mathrm{Mn}, \mathrm{Fe})_{2}(\mathrm{P}, \mathrm{Si})$ compound than in the $\mathrm{Fe}_{2} \mathrm{P}$ compound. As presented in Fig. 14, $\tau \propto \xi^{n}$ shows a power-law dependence on $\xi$ with an exponent $n=1.8(0.1)$. Comparing this scaling behavior with Eq. (6), it suggests that the ratio between $\int \frac{d \sigma}{d \Omega}(Q) d Q$ and $\lambda$ is weakly temperature dependent. With the neutron and muon data at hand, we have not extracted the correlation time and correlation length above $T_{C}$ for the

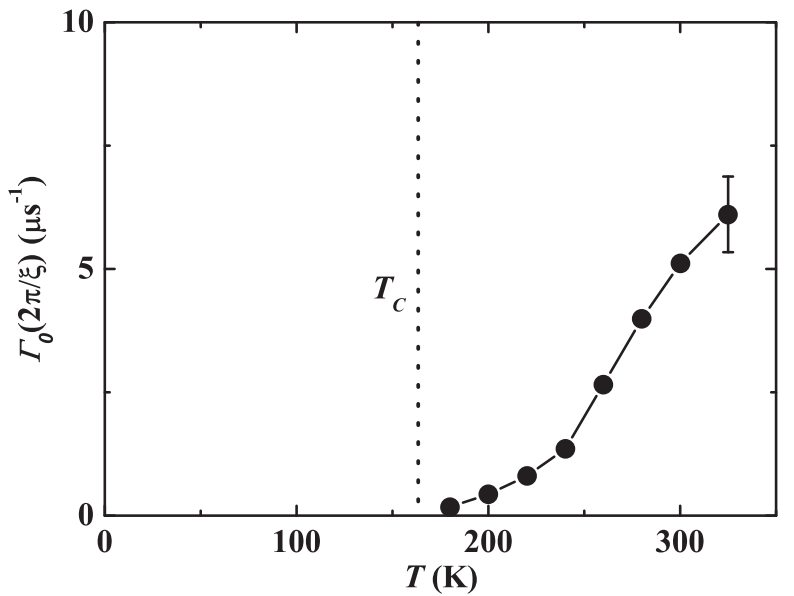

FIG. 13. Temperature dependence of the magnetic relaxation rate for $\mathrm{Mn}_{1.70} \mathrm{Fe}_{0.25} \mathrm{P}_{0.50} \mathrm{Si}_{0.50}$.

$\mathrm{Mn}_{1.00} \mathrm{Fe}_{0.95} \mathrm{P}_{0.67} \mathrm{Si}_{0.33}$ compound due to the appearance of the unexpected intermediate AFM phase.

\section{DISCUSSION}

The magnetoelastic transition in $(\mathrm{Mn}, \mathrm{Fe})_{2}(\mathrm{P}, \mathrm{Si})$ compounds is accompanied by a metamagnetic transition, i.e., a transition from a low-moment state to a high-moment state for the Fe atoms [2,7]. In contrast, the magnetic moment of the Mn atoms (about 2.6 $\mu_{B}$ ) is preserved when crossing the FM-PM transition [2,7]. The local magnetic field exerted on the $\mathrm{Fe}$ atoms by the $\mathrm{Mn}$ atoms triggers the moment formation and magnetic order [7,22]. Therefore, magnetic correlations between Mn atoms in the PM state will enhance the local magnetic field, promote the formation of Fe magnetic moment, and will finally result in long-range magnetic order.

The combination of polarized neutron diffraction and $\mu \mathrm{SR}$ experiments reveals the presence of short-range magnetic correlations in the $\mathrm{PM}$ state of $(\mathrm{Mn}, \mathrm{Fe})_{2}(\mathrm{P}, \mathrm{Si})$ compounds. This causes a deviation of the PM susceptibility from the

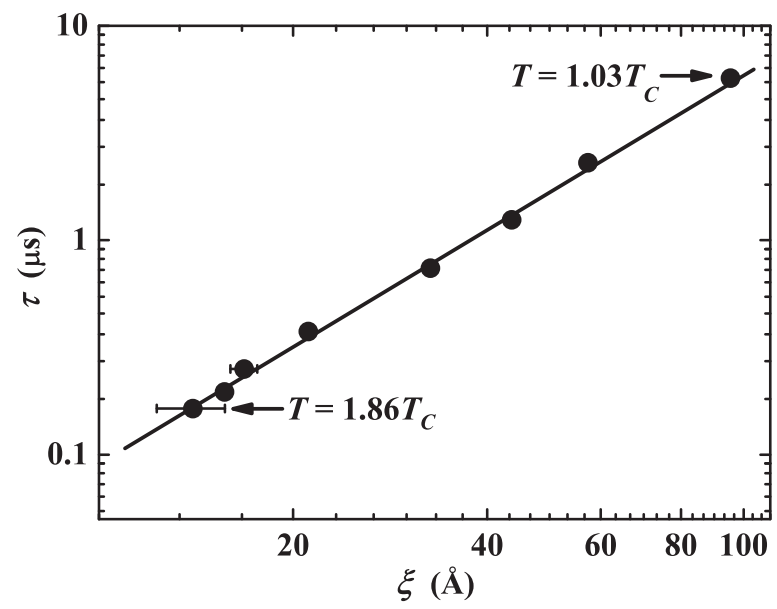

FIG. 14. Relation between the correlation time and correlation length for $\mathrm{Mn}_{1.70} \mathrm{Fe}_{0.25} \mathrm{P}_{0.50} \mathrm{Si}_{0.50}$. The solid line represents a fit to the data. 
Curie-Weiss law, since the molecular field approximation neglects the magnetic correlations in the PM state. The increasing correlation length and slowing down of the magnetic fluctuations with decreasing temperature reflects the enhanced magnetic correlations. Compared to $\mathrm{Fe}_{2} \mathrm{P}$, the $(\mathrm{Mn}, \mathrm{Fe})_{2}(\mathrm{P}, \mathrm{Si})$ compound shows stronger magnetic correlations in the paramagnetic state, which benefits the strong metamagnetic transition responsible for the giant magnetocaloric effect. Consequently, the formation and development of short-range magnetic correlations in the PM state plays a crucial role in the magnetoelastic transition and the resultant giant magnetocaloric effect in $(\mathrm{Mn}, \mathrm{Fe})_{2}(\mathrm{P}, \mathrm{Si})$ compounds.

\section{CONCLUSIONS}

$x y z$ neutron polarization and muon-spin relaxation studies on $(\mathrm{Mn}, \mathrm{Fe})_{2}(\mathrm{P}, \mathrm{Si})$ reveal, both on the length- and timescales, the presence of short-range magnetic correlations in the PM regime. The short-range magnetic correlations develop in space and slow down with decreasing temperature, and finally become long-range and static at $T_{C}$. This study provides a better understanding of the magnetoelastic phase transition in $\mathrm{Fe}_{2} \mathrm{P}$-type compounds.

\section{ACKNOWLEDGMENTS}

The authors thank Anton Lefering and Bert Zwart for their help with the sample preparation and acknowledge the technical assistance of Jouke Heringa for $\mu$ SR data analysis. Part of this work was performed at the Swiss Muon Source, Paul Scherrer Institut, Villigen, Switzerland. J.C. acknowledges the financial support from the Swedish Research Council. This work is a part of an Industrial Partnership Program IPP I28 of the Dutch Foundation for Fundamental Research on Matter (FOM), co-financed by BASFNew Business.
[1] X. F. Miao, L. Caron, P. Roy, N. H. Dung, L. Zhang, W. A. Kockelmann, R. A. de Groot, N. H. van Dijk, and E. Brück, Tuning the phase transition in transition-metal-based magnetocaloric compounds, Phys. Rev. B 89, 174429 (2014).

[2] N. H. Dung, Z. Q. Ou, L. Caron, L. Zhang, D. T. Cam Thanh, G. A. de Wijs, R. A. de Groot, K. H. J. Buschow, and E. Brück, Mixed magnetism for refrigeration and energy conversion, Adv. Energy Mater. 1, 1215 (2011).

[3] F. Guillou, G. Porcari, H. Yibole, N. H. van Dijk, and E. Brück, Taming the first-order transition in giant magnetocaloric materials, Adv. Mater. 26, 2671 (2014).

[4] M. J. Neish, M. P Oxley, J. Guo, B. C. Sales, L. J. Allen, and M. F. Chisholm, Local Observation of the Site Occupancy of Mn in a MnFePSi Compound, Phys. Rev. Lett. 114, 106101 (2015).

[5] X. F. Miao, L. Caron, Z. Gercsi, A. Daoud-Aladine, N. H. van Dijk, and E. Brück, Thermal-history dependent magnetoelastic transition in $(\mathrm{Mn}, \mathrm{Fe})_{2}(\mathrm{P}, \mathrm{Si})$, Appl. Phys. Lett. 107, 042403 (2015).

[6] Z. Q. Ou, L. Zhang, N. H. Dung, L. van Eijck, A. M. Mulders, M. Avdeev, N. H. van Dijk, and E. Brück, Neutron diffraction study on the magnetic structure of $\mathrm{Fe}_{2} \mathrm{P}$-based $\mathrm{Mn}_{0.66} \mathrm{Fe}_{1.29} \mathrm{P}_{1-x} \mathrm{Si}_{x}$ melt-spun ribbons, J. Magn. Magn. Mater. 340, 80 (2013).

[7] N. H. Dung, L. Zhang, Z. Q. Ou, L. Zhao, L. van Eijck, A. M. Mulders, M. Avdeev, E. Suard, N. H. van Dijk, and E. Brück, High/low-moment phase transition in hexagonal Mn-Fe-P-Si compounds, Phys. Rev. B 86, 045134 (2012).

[8] O. Beckman and L. Lundgren, in Handbook of Magnetic Materials, edited by K. H. J. Buschow (North-Holland, Amsterdam, 1991), Vol. 6, pp. 181-287.

[9] R. Zach, M. Guillot, and R. Fruchart, The influence of high magnetic fields on the first order magneto-elastic transition in $\operatorname{MnFe}\left(\mathrm{P}_{1-y} \mathrm{As}_{y}\right)$ systems, J. Magn. Magn. Mater. 89, 221 (1990).

[10] H. Yabuta, K. Umeo, T. Takabatake, K. Koyama, and K. Watanabe, Temperature- and field-induced first-order ferromagnetic transitions in $\operatorname{MnFe}\left(\mathrm{P}_{1-x} \mathrm{Ge}_{x}\right)$, J. Phys. Soc. Jpn. 75, 113707 (2006).

[11] H. Fujii, Y. Uwatoko, K. Motoya, Y. Ito, and T. Okamoto, Neutron scattering investigation of itinerant electron system $\mathrm{Fe}_{2} \mathrm{P}$, J. Phys. Soc. Jpn. 57, 2143 (1988).
[12] C. Wilkinson, R. Wäppling, and K. R. A. Ziebeck, Spin fluctuations in $\mathrm{Fe}_{2} \mathrm{P}$ above its Curie temperature, J. Magn. Magn. Mater. 78, 269 (1989).

[13] R. Wäppling, O. Hartmann, E. Wackelgard, and T. Sundqvist, $\mu$ SR study of $\mathrm{Fe}_{2} \mathrm{P}$ in the paramagnetic state, J. Magn. Magn. Mater. 50, 347 (1985).

[14] H. Yamada and K. Terao, First-order transition of $\mathrm{Fe}_{2} \mathrm{P}$ and anti-metamagnetic transition, Phase Transit. 75, 231 (2002).

[15] O. Schärpf and H. Capellmann, The xyz-difference method with polarized neutrons and the separation of coherent, spin incoherent, and magnetic scattering cross sections in a multidetector, Phys. Status Solidi A 135, 359 (1993).

[16] J. R. Stewart, P. P. Deen, K. H. Andersen, H. Schober, J. F. Barthélémy, J. M. Hillier, A. P. Murani, T. Hayes, and B. Lindenau, Disordered materials studied using neutron polarization analysis on the multi-detector spectrometer, D7, J. Appl. Cryst. 42, 69 (2009).

[17] G. Ehlers, J. R. Stewart, A. R. Wildes, P. P. Deen, and K. $\mathrm{H}$. Andersen, Generalization of the classical xyz-polarization analysis technique to out-of-plane and inelastic scattering, Rev. Sci. Instrum. 84, 093901 (2013).

[18] J. Rodríguez-Carvajal, Recent advances in magnetic structure determination by neutron powder diffraction, Physica B 192, 55 (1993).

[19] N. H. Dung, L. Zhang, Z. Q. Ou, and E. Brück, From first-order magneto-elastic to magneto-structural transition in $(\mathrm{Mn}, \mathrm{Fe})_{1.95} \mathrm{P}_{0.50} \mathrm{Si}_{0.50}$ compounds, Appl. Phys. Lett. 99, 092511 (2011).

[20] $T_{C}$ is defined as the temperature where the first derivative of susceptibility with respect to the temperature reaches a maximum.

[21] X. F. Miao, Y. Mitsui, A. Iulian Dugulan, L. Caron, N. V. Thang, P. Manuel, K. Koyama, K. Takahashi, N. H. van Dijk, and E. Brück (unpublished).

[22] E. K. Delczeg-Czirjak, M. Pereiro, L. Bergqvist, Y. O. Kvashnin, I. Di Marco, G. Li, L. Vitos, and O. Eriksson, Origin of the magnetostructural coupling in $\mathrm{FeMnP}_{0.75} \mathrm{Si}_{0.25}$, Phys. Rev. B 90, 214436 (2014) 
[23] N. H. Dung, L. Zhang, Z. Q. Ou, and E. Brück, Magnetoelastic coupling and magnetocaloric effect in hexagonal Mn-Fe-P-Si compounds, Scr. Mater. 67, 975 (2012).

[24] T. J. Hicks, Magnetism in Disorder, Oxford Series on Neutron Scattering in Condensed Matter (Oxford University Press, New York, 1995).

[25] S.W. Lovesey, Theory of Neutron Scattering from Condensed Matter, International Series of Monographs on Physics, Vol. 2 (Clarendon Press, Oxford, 1984).

[26] G. Caglioti, A. Paoletti, and F. P. Ricci, Choice of collimators for a crystal spectrometer for neutron diffraction, Nucl. Instr. 3, 223 (1958).

[27] A. W. Hewat, Design for a conventional high-resolution neutron powder diffractometer, Nucl. Instrum. Methods 127, 361 (1975).

[28] R. Kubo and T. Toyabe, A stochastic model for low field resonance and relaxation, in Magnetic resonance and relaxation, edited by R. Blinc (North-Holland, Amsterdam, 1967), p. 810.
[29] R. S. Hayano, Y. J. Uemura, J. Imazato, N. Nishida, T. Yamazaki, and R. Kubo, Zero- and low-field spin relaxation studied by positive muons, Phys. Rev. B 20, 850 (1979).

[30] A. Keren, Generalization of the abragam relaxation function to a longitudinal field, Phys. Rev. B 50, 10039 (1994).

[31] P. Dalmas de Réotier and A. Yaouanc, Quantum calculation of the muon depolarization function: effect of spin dynamics in nuclear dipole systems, J. Phys.: Condens. Matter 4, 4533 (1992).

[32] A. Yaouanc and P. Dalmas de Réotier, Muon Spin Rotation, Relaxation, and Resonance: Applications to Condensed Matter, International Series of Monographs on Physics (Oxford University Press, New York, 2011).

[33] F. Mezei, Critical dynamics in isotropic ferromagnets, J. Magn. Magn. Mater. 45, 67 (1984). 